\title{
FRET-assisted organic dye molecules using in conjunction with colloidal quantum dots for bio-labelling
}

\author{
Evren Mutlugun and Hilmi Volkan Demir*
}

\begin{abstract}
Department of Physics, Department of Electrical and Electronics Engineering, and UNAM - Institute of Materials Science and Nanotechnology, Bilkent University, TR-06800, Ankara, Turkey; Nanyang Technological University, School of Electrical and Electronic Engineering, Division of Microelectronics, School of Physical and Mathematical Sciences, Luminous! Semiconductor Lighting and Display Center of Excellence, Nanyang Avenue, Singapore 639798, Singapore *e-mail: volkan@stanfordalumni.org
\end{abstract}

Organic dye molecules are commonly used for labeling in biological applications since they possess high quantum yield and good thermal- and photo-stability [1]. Although their molecular size allows them to be used widely for various bio-labeling applications, their intrinsic material properties prevent them to be used spectrally in a more efficient way. One of the main factors that limit the use of these dye molecules is their narrow absorption spectra. These dye molecules can only be optically pumped efficiently in a characteristically narrow spectrum. This prevents the possibility of multiple labeling of biomolecules with a single source to excite different color dyes at the same time (also known as spectral multiplexing). To solve this problem, we propose and demonstrate light-harvesting quantum dots to excite organic dyes via Förster-type resonance energy transfer (FRET), which effectively extends absorption of dye molecules spectrally and enhances their emission intensity. Although nanocrystals have previously been used for energy transfer agents to dye molecules in our group and the others [2-4], the enhancement of the emission intensity and light-harvesting of acceptors have not been studied for quantum dot-Atto dye pairs. Such organic Atto dyes are widely used in bioapplications such as labeling and tagging [5-6].

In this work, for this purpose, organic Atto dye molecules are FRET-coupled with quantum dot light-harvesters. We synthesize organic CdSe quantum dots, emitting at $584 \mathrm{~nm}$ with a quantum yield of $31 \%$ (which are carefully selected to spectrally match the absorption band of the acceptor dye molecules) (Fig.1), using hot injection method. To transfer the excitation energy of the donor CdSe quantum dots, we use cationic Atto Rhomdamine 12 molecules as acceptors of which their positive net charge provides a good match with the slightly negatively charged CdSe quantum dots, allowing them to electrostatically attract towards each other in the solution. By changing the acceptor to donor concentration ratio in solution, namely from 0 to 8.6, we show the light-harvesting enhancement of the steady state photoluminescence - SSPL (with a factor of up to 4 folds), together with the lifetime modifications of the donor and acceptor molecules verifying FRET based energy transfer.

The steady state photoluminescence measurements are first performed at $375 \mathrm{~nm}$ excitation wavelength (to match the emission wavelength of the pulsed laser diode head used in the time resolved measurement - TRPL), and the light harvesting factor is calculated using the overall photoluminescence profile of the donor and acceptor molecules compared to the bare donor and bare acceptor contributions, as a function of A/D ratio. The corresponding enhacement factor of the acceptor dye molecule when excited at $375 \mathrm{~nm}$ is presented in Fig. 1, with a maximum level of up to 2.8. In addition to that, to demonstrate the advantage of using quantum dots as energy transfer agents to organic dye molecules, we set the steady state excitation wavelength at $466 \mathrm{~nm}$, which corresponds to weak excitation and absorption of the organic dye molecules, compared to strong absorbance of the CdSe quantum dots (Fig. 1). The light harvesting factor of the accepor molecule analysed from SSPL measurement when excited at 466nm is also shown in Fig. 1. This is found out to be providing up to 4 times light enhancement.

To demonstrate the lifetime modifications of the donor and acceptor pairs, we also perform time-resolved fluorescence measurements using the PicoQuant FluoTime 200 system. Fig. 2 shows the evolution of the timeresolved decay of the donor molecule as acceptor to donor concentration ratio is varied. The analysis of the shortening of the donor lifetime and the increase of the acceptor lifetime is also given in Fig.2. To prevent the mixing of the tails of the emission of the donor and acceptor molecules, TRPL measurements are performed far from the acceptor molecule emission tail, at 560nm for donor lifetime analysis; and far from donor emission tail, at $650 \mathrm{~nm}$ for acceptor lifetime analysis.

In conclusion, we demonstrated FRET-meadiated light-harvesting of CdSe quantum dots for Atto Rhodamine 12 dye molecules using selective excitation wavelenghts and achieved up to 4-fold enhancement of the Atto emission. We showed strong lifetime modifications of the donor and acceptor molecules as a result of lightharvesting FRET. These results indicate that such FRET-assistded light-harvesting will be important for biolabeling. 
Acknowledgements: This work is supported by NRF-RF 2009-09, EU-FP7 Nanophotonics4Energy NoE, and TUBITAK EEEAG 107E088, 109E002, 109E004, and 110E010. HVD acknowledges support from ESF-EURYI and TUBA-GEBIP, and EM, from TUBITAK-BIDEB.

References

[1] Y. Li, et.al, "Multiplexed detection of pathogen DNA with DNA- based fluorescence nanobarcodes," Nature Biotech. 23, 885-889 (2005).

[2] E. Mutlugun, et.al , "Highly efficient FRET based light harvesting using aqueous CdTe quantum dot antennas", Optics Express 18, 10720$10730(2010)$

[3] E. Mutlugun, et. al, "Highly efficient nonradiative energy transfer using charged CdSe/ZnS nanocrystals for light-harvesting in solution," Applied Physics Letters 95, 033106 (2009).

[4] A. R. Clapp, et.al , "Förster resonance energy transfer investigations using quantum dot fluorophores," Chem. Phys. Chem. 7, 47-57 (2006).

[5] E. A. Jares-Erijman, et.al, "FRET imaging," Nature Biotech. 21, 1387-1395 (2003).

[6]M. T. F Arguelles, et.al, "Synthesis and Characterization of Polymer-Coated Quantum Dots with Integrated Acceptor Dyes as FRET-Based Nanoprobes", Nano Letters 7, 2613-1617 (2007).
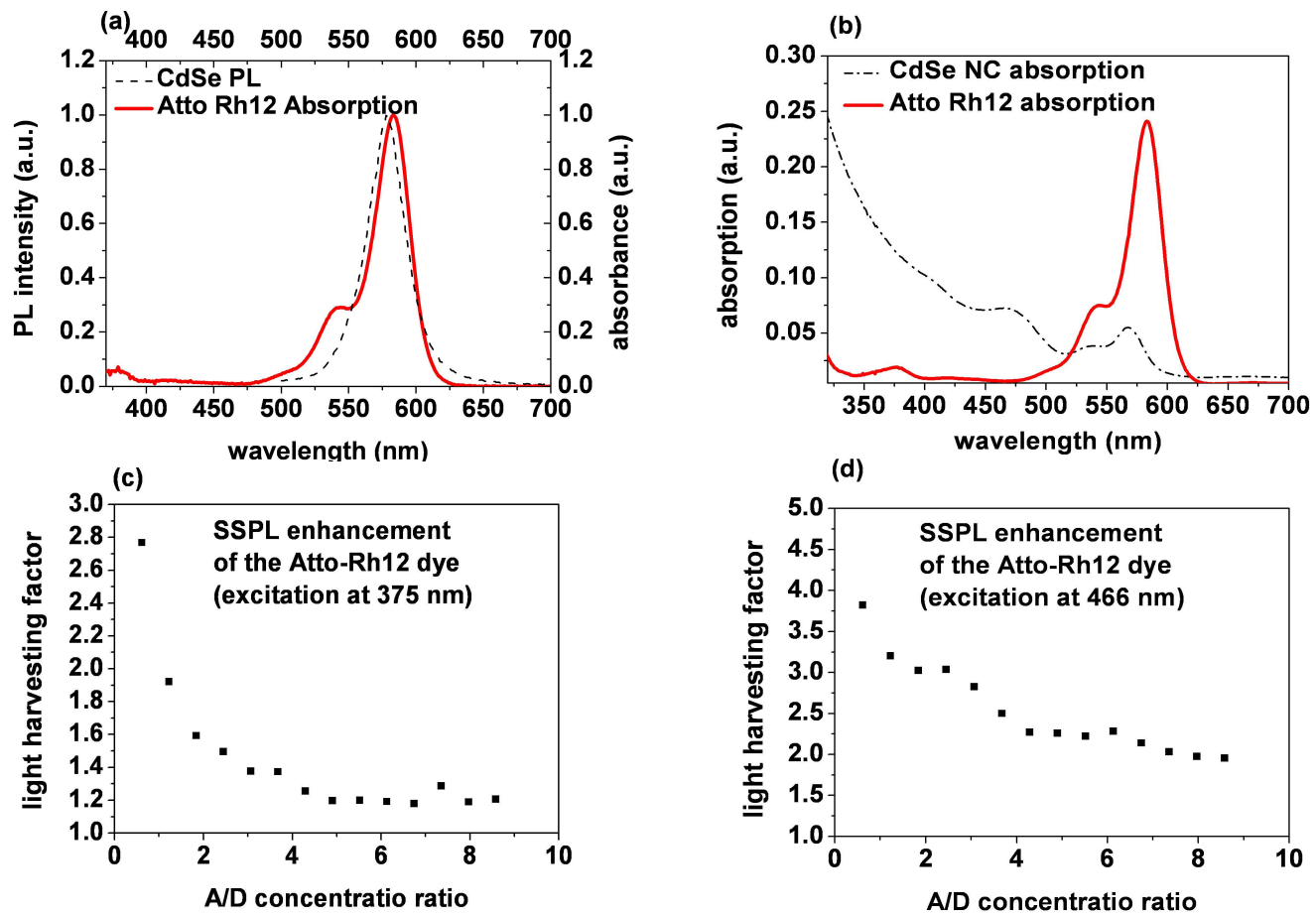

Figure 1. (a) Photoluminescence spectrum of CdSe quantum dots together with the absorption profile of the acceptor dye molecules. (b) Absorption spectrum of the CdSe quantum dots and the acceptor dye molecules. (c) Light harvesting factor for the acceptor molecules extracted from SSPL enhancement when excited at $375 \mathrm{~nm}$ and (d) when excited at $466 \mathrm{~nm}$, both parameterized with respect to varied A/D concentration ratios.
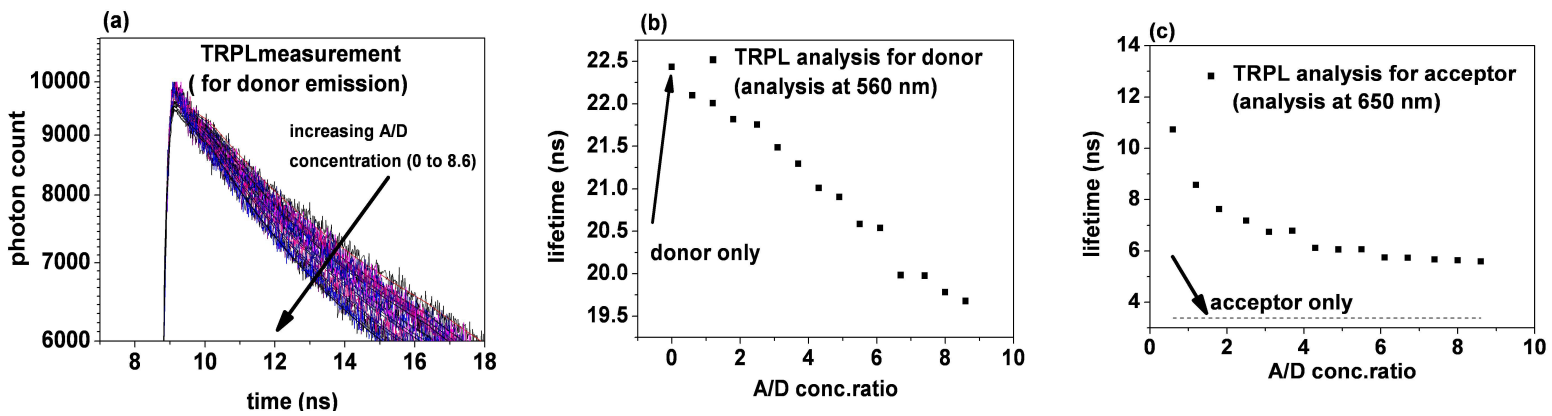

Figure 2. (a) TRPL decays for donor CdSe nanocrystals, (b) lifetime analysis of the donor molecules (at 560nm), and (c) lifetime analysis of acceptor molecules (at $650 \mathrm{~nm}$ ), all as a function of $\mathrm{A} / \mathrm{D}$ ratio. 